\title{
Psychiatry for the General Practitioner Trainee
}

\author{
(from a special correspondent)
}

Should the future GP do a six months' senior house officer job in psychiatry as part of his or her training? This was the central question at a one-day discussion held on 28 May 1980 at the Royal College of General Practitioners in Princes Gate, London, following the publication of the guidelines prepared by a RCPsych/RCGP Liaison Committee (Bulletin, June 1980, p 93) and presented to the conference by Dr Thomas Bewley. As Professor N. Kessel, among others, pointed out, psychiatric hospitals and units stand to gain considerably if they can recruit these doctors to their junior staff. Regular SHO posts are often difficult to fill at present, and GP trainees may be of very good quality. They do not expect to compete up the specialty training ladder, but they will carry part of the work-load. On the other hand, is what the hospital offers what they really need, or can it be made so?

Dr Anthony Clare rehearsed the evidence from general practice. One in seven of all GP consultations involves mental health, the average GP sees about 300 patients a year with anxiety and depression, and less than 2 per cent of all his cases of depression are referred on to the psychiatrist. On the other hand, the International Classification of Diseases does not fit his diagnostic needs, because only a small part of his work touches formal psychiatric illness and the greater part consists of the psychiatric associated disordersphysical ills with psychological complaints, often in a setting of social pathology. Formal psychiatry is a specialty on its own, but the wider psychiatry is a part of social medicine, and community mental health is bound to be looked after by generalists.

Dr Michael Courtenay, as a general practitioner, tried to analyse his psychiatric work. Patients referred themselves with symptoms (in many depressions these were somatic) but what was the prevalence and severity of these in the whole population, and what decided the individual to consult? One needed to know how to elucidate and also the role of threatening life events; one needed to understand the lure of the sick role, the patterns of family culture, minority ways of life such as skid row, and the large area of sexual problems. The GP needed to know how to take a full psychiatric history, and also to be made aware of his own psychodynamics. He had to learn the art of communication. Although arranging psychiatric hospital admission could be a matter of life or death, or be of grave import to both patient and family, it was a very small part of the GP's total work. One member of the audience in fact remarked that he signed a Mental Health Recommendation about once a year.

In the following discussion it was made clear that modern undergraduate training in psychiatry was quite insufficient for practice, but it seemed to be widely felt that psychiatrists, and also psychologists, social workers and sociologists, had something useful to offer the trainee. The fear was that the average SHO post offered only in-patient work with acute psychoses and with chronic patients when what was needed was out-patient work, exposure to child psychiatry, and (as Dr D. H. Dick of the Hospital Advisory Service pointed out) experience of the organizational side of the psychiatry of old age rather than simply of the diagnosis of dementia. This raised the need to vet posts beforehand to see if they were suitable for trainees, and to provide some ongoing supervision by a tutor. Criteria for approval might be different from those employed by the Royal College of Psychiatrists for specialty training. Who should approve, whether centrally or locally; and who should act as tutor?

Dr John Horder (President, RCGP) wanted to know why all SHOs, whether future GPs or future consultants, could not have the same initial training, but Professor Kessel was very firm that the training for specialism was different: he did not believe any psychiatric beginner should see out-patients until he had mastered the skills of taking history and mental state in his first six months, working only with in-patients. On the other hand he felt the GP trainee ought to have longer than six months of psychiatry because so many clinical problems ran on over years. He suggested the trainee should continue supervision under regular guidance for a further year of all the in-patients he had treated while an SHO. Dr R. M. Berrington surveyed non-hospital ways of meeting the GP's need for psychiatric training, by sessional clinical assistantships, special attachments, and half-day release schemes. Special matters, such as awareness and use of the doctor-patient relationship, could be developed by special groups, on the Balint model, and by keeping a training diary or pursuing a specially chosen learning project. This might help to meet the trainees' wider needs which hospital experience could not satisfy, but in any case there would not on present showing be enough SHO posts for every trainee to have one.

Three recent trainees spoke about their experiences. One had enjoyed (like his 12 SHO predecessors and colleagues) working in a district general hospital psychiatric unit which was community-orientated and practised crisis intervention. This had given great confidence in handling psychiatric patients, a knowledge of community services, and some understanding of group dynamics, but was lacking in medical model psychiatric teaching, and teaching about psychotropic drugs which the GP certainly needed. Another, working in a more orthodox 700-bed psychiatric hospital, had been fortunate in his consultant and able to shape his week's work so that it included two sessions of out-patients (where he saw new patients, and the consultant the follow- 
ups), one session on rehabilitation of alcoholics and chronic patients, and one session at another hospital doing child psychiatry. He was also able to undertake all the self-poisoning assessments and liaison psychiatry. Most of his fellow trainees in the Region complained bitterly that they only treated a heavily psychotic and custodial care population, which was poor preparation for future practice.

Most participants seemed to agree that the recommendations (Bulletin, June, p 93) were on the right lines, though not the whole story; and that a period of hospital psychiatric experience should be very valuable, not least in showing how the psychiatric services work. But psychiatrists might benefit from knowing how general practice works, and there was some support for the suggestion, which will be further explored, that six months in general practice should be part of the experience of every psychiatric specialty trainee. Such an option or requirement would of course release more junior hospital posts, and possibly enable all instead of only some GP trainees to get their six months in a psychiatric hospital.

\section{Reviews}

Prevention in Mental Heallh. MIND Annual Conference, London, October 1979. 119 pp. £2.95.

Prevention has for a long time ceased to be the subject of much enthusiasm on the part of psychiatrists. Caution seems to stem particularly from doubts about its feasibility, a concern that resources should not be syphoned away from much needed treatment of established illness, or reservations about the relevance of psychosocial stress to mental illness, especially when the latter is defined in biomedical disease entity terms. Nevertheless, this report contains a great deal which is relevant to clinical psychiatry and demands to be taken very seriously. It looks at mental health care problems primarily from a socio-economic perspective, examines the current community setting of mental distress, describes many exciting new initiatives, and gives special attention to high risk population sub-groups. The report consists of four sections concerned with mental health in the community and in the work place, the mental health of women and children, and various aspects of childbirth and infant care.

The scene is set by two main introductory position papers. Peter Draper issues the challenge that treaters are not necessarily the best preventers, because the necessafy skills do not overlap. Health is therefore not to be equated with the establishment of more treatment services. He also insists that resistance to prevention may stem from complex motives which are not always altruistic: the way forward must depend on genuine socio-political changes rather than 'medico-legal heroes,' this being the note of mock respect which he reserves for the Victorian MOH. Fortunately the temperature of the report then falls to a more tolerable level, at least one which promotes a constructive dialogue, with Anthony Clare's well balanced look at feasibility: he warns of overweening enthusiasm for particular approaches and their misapplication, and emphasises the need to distinguish between morality and health and indeed to define a clear remit. Yet we must reflect at this stage that neither of those two giants of preventive medicine, Snow and Goldberger, knew the cause of cholera and pellagra when they efiectively demonstrated how to prevent them. They won through because of an astonishing zeal for accurate observation, persistence, and thoroughness in applying simple techniques. The unsophisticated approach, and indeed the intuitive one, may well be worth backing. We need also to remember basic issues: as the report proceeds doctors are urged by Tony Smythe to be more on the side of their patients, to show less professional arrogance, and to foster early intervention in a non-threatening way.

'Mental Health in the Community' considers the American experience of community mental health centres and the controversy over their effectiveness, as well as special initiatives which have developed in this country. The battle of the mental hospital versus the community service is engaged, but not in a way which provides sufficient hard evidence to decide which side wins in the end. The role of the psychiatrist is challenged by Roger Hargreaves of the Brindle House Mental Health Centre, near Manchester, where diversion of clients away from the formal psychiatric service is encouraged in order to avoid labelling and unnecessary medication. We must pause here: are we psychiatrists doing people more harm than good? Are we to be pensioned off to the mental hospitals to reign supreme over our ECT boxes and psychotropic drugs? Will society ultimately only allow us a role in tertiary prevention? The report then moves on to the problem of immigrant groups in Bradford, where Dr Rack's initiative as a psychiatrist is gratefully acknowledged, followed by several shorter but refreshing contributions on community psychiatric nursing, work with the physically handicapped, and day centres.

'Mental Health in the Work Place' constitutes the second main section of this report. Elliot Stern's paper provides a startling insight into the stresses at work today, where the deskilling and loss of jobs inherent in the introduction of 